\title{
A stem cell marker KLF5 regulates CCAT1 via three-dimensional genome structure in colorectal cancer cells
}

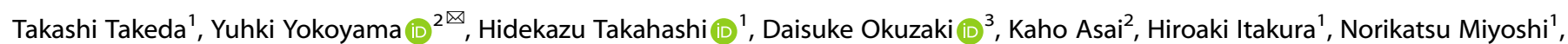 \\ Shogo Kobayashi ${ }^{1}$, Mamoru Uemura ${ }^{1}$, Toshitsugu Fujita ${ }^{4}$, Hiroo Ueno ${ }^{5}$, Masaki Mori ${ }^{6}$, Yuichiro Doki ${ }^{1}$, Hodaka Fujii ${ }^{4}$, \\ Hidetoshi Eguchi ${ }^{1}$ and Hirofumi Yamamoto ${ }^{1,2}$
}

(c) The Author(s) 2021

BACKGROUND: KLF5 plays a crucial role in stem cells of colorectum in cooperation with Lgr5 gene. In this study, we aimed to explicate a regulatory mechanism of the KLF5 gene product from a view of three-dimensional genome structure in colorectal cancer (CRC).

METHODS: In vitro engineered DNA-binding molecule-mediated chromatin immunoprecipitation (enChIP)-seq method was used to identify the regions that bind to the KLF5 promoter.

RESULTS: We revealed that the KLF5 promoter region interacted with the KLF5 enhancer region as well as the transcription start site (TSS) region of the Colon Cancer Associated Transcript 1 (CCAT1) gene. Notably, the heterodeletion mutants of KLF5 enhancer impaired the cancer stem-like properties of CRC cells. The KLF5 protein participated in the core-regulatory circuitry together with co-factors (BRD4, MED1, and RAD21), which constructs the three-dimensional genome structures consisting of KLF5 promoter, enhancer and CCAT1 TSS region. In vitro analysis indicated that KLF5 regulated CCAT1 expression and we found that CCAT1 expression was highly correlated with KLF5 expression in CRC clinical samples.

CONCLUSIONS: Our data propose the mechanistic insight that the KLF5 protein constructs the core-regulatory circuitry with cofactors in the three-dimensional genome structure and coordinately regulates KLF5 and CCAT1 expression in CRC.

British Journal of Cancer (2022) 126:109-119; https://doi.org/10.1038/s41416-021-01579-4

\section{BACKGROUND}

A zinc finger transcription factor Krüppel-like factor 5 (KLF5) plays an important role in the stemness of embryonic stem (ES) cells $[1,2]$ and developmental processes of respiratory epithelium, bladder epithelium, mammary gland and ocular surface [3-5]. In the intestinal epithelium, KLF5 is suggested to play a role in the maintenance of intestinal stem cells and their niche $[6,7]$.

KLF5 has been reported as a lineage-survival oncogene whose expression is upregulated in specific cancer types, such as squamous carcinoma (e.g., head and neck cancer, oesophageal cancer) and gastrointestinal cancer (e.g., colorectal cancer, gastric cancer, pancreatic cancer) [8]. Cancer stem cells (CSCs) are defined as a subpopulation of cancer cells with a self-renewal capacity and multilineage potency and considered a source of tumour recurrence and metastasis [9]. KLF5 is associated with CSC-like properties because KLF5 knockdown suppressed sphere-formation activity in colorectal cancer (CRC) cell lines
[10]. We also reported that miR-4711-5p, which directly targets the 3'-untranslated regions (3'-UTRs) of KLF5, suppressed CSC properties in CRC cell lines [11]. Moreover, KLF5 deletion prevented the tumourigenesis of $\mathrm{Lgr}^{+}$intestinal stem cells induced by the mutated $\beta$-catenin gene [12].

Although the underlying mechanism of how the KLF5 gene product is expressed in CRC has not been clarified, one of the mechanisms might be due to the three-dimensional genome structure of the KLF5 gene. Cell-type-specific gene expression is occasionally regulated by $3 \mathrm{D}$ genome structure, as represented by promoter-enhancer looping. It is reported that promoter-enhancer looping takes place when cells undergo differentiation in developmental processes, leading to essential gene expression [13, 14]. In instances, the gene-activation element called the locus control region (LCR) interacts with the $\gamma$-globin gene in fetuses, and its interaction is switched to the $\beta$-globin gene in adults [15]. We also previously demonstrated that the CSC-related gene $A L D H 1 A 1$ was regulated by

\footnotetext{
${ }^{1}$ Department of Surgery, Gastroenterological Surgery, Graduate School of Medicine, Osaka University, 2-2, Yamadaoka, Suita, Osaka 565-0871, Japan. ${ }^{2}$ Department of Molecular Pathology, Division of Health Sciences, Graduate School of Medicine, Osaka University, 1-7, Yamadaoka, Suita, Osaka 565-0871, Japan. ${ }^{3}$ Single Cell Genomics, Human Immunology, WPI Immunology Frontier Research Center, Osaka University, 3-1, Yamadaoka, Suita, Osaka 565-0871, Japan. ${ }^{4}$ Department of Biochemistry and Genome Biology, Graduate School of Medicine, Hirosaki University, 5 Zaifu-cho, Hirosaki, Aomori 036-8562, Japan. ${ }^{5}$ Department of Stem Cell Pathology, Kansai Medical University, 2-5-1 Shin-machi, Hirakata, Osaka 573-1010, Japan. ${ }^{6}$ School of Medicine, Tokai University, 143 Shimokasuya, Isehara, Kanagawa 259-1193, Japan. email: yyokoyama@sahs.med.osaka-u.ac.jp
} 
BRD4-dependent promoter-enhancer looping in ovarian cancer [16]. It is well known that the BRD4 as well as mediator complex and cohesin complex play a crucial role in the construction of 3D genome interaction [17]. MED1 and RAD21 are one of the components of the mediator complex and cohesin complex, respectively $[18,19]$.

Enhancers regulate gene expression by interacting with a promoter and are marked by histone modifications, such as acetylation at lysine 27 of histone H3 (H3K27ac) and monomethylation at lysine 4 of histone H3 (H3K4me1). In an effort to identify the KLF5 enhancer region in CRC cells, we used in vitro engineered DNA-binding molecule-mediated chromatin immunoprecipitation (enChIP) combined with next-generation sequencing (NGS) (in vitro enChIP-seq) $[20,21]$. In addition to the enhancer region, we found that the KLF5 promoter region interacted with a long noncoding RNA, the Colon Cancer Associated Transcript 1 (CCAT1). CCAT1 is a long noncoding RNA that was initially found to be upregulated in CRC [22], and studies have reported that high CCAT1 expression is associated with poor prognosis in CRC patients [23]. Subsequently, overexpression of CCAT1 was reported in various cancer types, such as gastric cancer and oesophageal cancer [24-26]. CCAT1 functions as a sponge for miRNAs [27], thereby contributing to malignant features of CRC by promoting cell proliferation, invasion and drug resistance [28, 29]. Finally, we show that the KLF5 protein constructs the core-regulatory circuitry with cofactors in the three-dimensional genome structure involving KLF5 gene and CCAT1.

\section{METHODS}

\section{Cell lines and cell culture}

Human CRC cell lines HT29 and SW48 were purchased from the American Type Culture Collection. Cells were cultured at $37^{\circ} \mathrm{C}$ in $5 \% \mathrm{CO}_{2}$. The mycoplasma test was performed prior to experiments. The KLF5 inhibitor ML264 [30] was purchased from Sigma Aldrich (St. Louis, MO, USA).

\section{Clinical tissue samples}

Clinical tissue samples for RNA extraction were collected from CRC patients $(n=131)$ who underwent surgery at the Osaka University hospital from 2003 to 2005. For the immunohistochemistry and in situ hybridisation, tissue samples were collected from CRC patients $(n=27)$ who underwent surgery from April 2019 to June 2019. Informed consent signatures were obtained from all patients. This study was approved by the Ethics Committee of Osaka University Hospital (No. 15144).

\section{Real-time quantitative PCR (qRT-PCR)}

The total RNA was extracted from cell lines and tissue samples by using the RNeasy Kit (QIAGEN, Hilden, Germany). qRT-PCR was performed as previously reported [11]. The sequences of the primers are listed in Supplementary Table S1.

\section{siRNA transfection}

siRNAs for BRD4 and negative control were purchased from GeneDesign (Osaka, Japan). siRNAs for MED1, RAD21 and KLF5 were purchased from Thermo Fisher Scientific (Waltham, MA, USA). siRNAs for CCAT1 were purchased from GeneDesign and Thermo Fisher Scientific. siRNA ( $50 \mathrm{nM}$ ) was transfected into CRC cell lines $24 \mathrm{~h}$ after seeding with Lipofectamine RNAiMAX reagent (Thermo Fisher Scientific). For the triple-knockdown experiment, siRNAs $(30 \mathrm{nM})$ for BRD4, MED1 and RAD21 were mixed and transfected into CRC cell lines. As a control, $90 \mathrm{nM}$ of negative control (NC) siRNA was transfected into CRC cell lines. The sequences of the siRNAs are listed in Supplementary Table S1.

\section{Cell-proliferation assay}

Cells were seeded in 96-well plates at a density of 4000 cells per well, and 5 -FU or oxaliplatin was added $24 \mathrm{~h}$ after seeding. Three days later, cell viability was evaluated by using Cell Counting Kit-8 solution (Dojindo Laboratories, Kumamoto, Japan).

\section{Sphere-formation assay}

Sphere-formation assay was performed as previously described [11]. At 3 weeks after seeding, the number of spheres $(\geq 50 \mu \mathrm{m})$ in all wells was counted.

\section{Flow cytometry analysis}

Cells were washed with PBS containing $2 \%$ FBS and incubated with primary antibody (Ab), anti-CD133/1 (AC133)-APC (Miltenyi Biotec, Bergisch Gladbach, Germany) or anti-CD44v9 (Cosmo Bio, Tokyo, Japan) at $4{ }^{\circ} \mathrm{C}$ for 20 min. For CD44v9, PE mouse anti-rat IgG2a (Becton, Dickinson and Company, Franklin Lakes, NJ, USA) was used as a secondary Ab. The data were analysed by using the SA3800 spectral cell analyser (Sony, Tokyo, Japan).

\section{Immunohistochemical staining}

Immunohistochemical staining was performed as described previously [31]. Rabbit polyclonal Ab for KLF5 (Sigma Aldrich) was used as a primary Ab. All specimens were evaluated individually by four researchers.

\section{RNA scope}

Paraffin-embedded tumour tissue samples were sectioned into $4-\mu \mathrm{m}$ sections and CCAT1 signals were detected using the Hs-CCAT1 target probe (Advanced Cell Diagnostics, Newark, CA, USA) and subsequently stained with RNA scope ${ }^{\circ}$ [32] (Advanced Cell Diagnostics).

\section{ChIP-qPCR}

ChIP experiment was performed as previously reported [33]. Cells were fixed and sheared by using Covaris M200 (Covaris, Woburn, MA, USA). The fragmented chromatin was incubated with the following primary Abs; KLF5 (Abcam, Cambridge, UK), MEIS1 (Abcam), RHOXF1 (GeneTex, Irvine, CA, USA), ZNF354C (Abcam), BRD4 (Bethyl Laboratories, Montgomery, TX, USA), MED1 (Bethyl Laboratories), RAD21 (Abcam). The Ab against normal rabbit IgG (Cell Signaling Technology, Danvers, MA, USA) was used as a negative control. The purified DNA was subjected to GPCR. GPCR was performed as described in the qRT-PCR section. The sequences of the primers are listed in Supplementary Table S1.

\section{Deletion of the genomic region by CRISPR/Cas9}

The Cas9 expression plasmid, hCas9 (a gift from Dr. George Church, Addgene, \#41815; http://n2t.net/addgene:41815; RRID:Addgene_41815) [34] gRNA Cloning Vector Bbsl ver. 2 plasmid (Addgene, \#85586; http:// n2t.net/addgene:85586; RRID:Addgene_85586) [35] and pEGFP-N3 (Clontech Laboratories, Mountain View, CA, USA) were used for the experiment. The synthesised nucleotides were annealed and cloned into the gRNA Cloning Vector Bbsl ver.2 plasmid.

HT29 cells were transfected with the hCas9 plasmid and two gRNA expression plasmids that target both ends of the KLF5 enhancer or CCAT1 TSS region, and pEGFP-N3 with Lipofectamine 3000 (Thermo Fisher Scientific). The next day, GFP-positive cells were sorted and seeded individually in 96 -well plates.

\section{In vitro enChIP-seq and bioinformatics analysis}

In vitro enChIP-seq was performed as previously reported [21]. Guide RNAs for the KLF5 promoter (gRNA-A, B) were designed by using the CRISPRdirect Web tool (https://crispr.dbcls.jp/). As a negative control, gRNA, which is designed in the promoter region of the chicken Pax5 gene, was used [21]. The crRNAs and tracrRNAs were synthesised by FASMAC (Kanagawa, Japan). The purified DNA was subjected to qPCR or NGS. The in vitro enChIP-seq library was prepared by using the TruSeq ChIP Library Preparation Kit (Illumina, San Diego, CA, USA). The libraries were sequenced as 36-bp single-end reads on the HiSeq3000 (Illumina). Images of NGS peaks were generated using Integrative Genomics Viewer (IGV) (http://software.broadinstitute.org/software/igv/ ). The sequences of the gRNAs and primers are listed in Supplementary Table S1.

\section{Statistical analysis}

Data are shown as the mean \pm SD. The data were compared by Student's $t$ test, chi-square test, or Fisher's exact test. The Kaplan-Meier method and log-rank test were used to calculate significant differences in patient survival. A value of $P<0.05$ was considered statistically significant. All 
statistical analyses were performed using Microsoft Excel or JMP statistical software (SAS Institute Inc., Cary, NC, USA).

\section{RESULTS}

Identification of the enhancer region for the KLF5 gene

To identify the enhancer region that binds to the KLF5 promoter, we employed an in vitro enChIP-seq method [20] (Supplementary Fig. S1A). The binding DNA sequence was analysed by NGS using two guide RNAs (gRNA-A, gRNA-B) designed 400-600 bp upstream of the transcription start site in the KLF5 promoter region (Supplementary Fig. S1B).

Compared with the negative control gRNA against the chicken Pax5 gene [21], several DNA sequences had a significant increase in binding to the gRNA-marked KLF5 promoter regions. As candidates for the $K L F 5$ enhancer, we first searched the genomic regions on chromosome 13 where KLF5 promoter is located (Fig. $1 \mathrm{a}$ and Supplementary Fig. S2A). Among them, we focused on Chr 13; 74,003,796 - 74,004,073 (P score rank \#3rd, Fig. 1a) located $a \sim 370 \mathrm{~kb}$ downstream of the KLF5 promoter rather than the other two regions with superior $P$ scores (rank \#1st and \#2nd) because the peaks of well-established enhancer markers such as histone H3 lysine 27 acetylation (H3K27ac) and BRD4 were robustly observed at this region compared with \#1st and \#2nd regions by the ChIP-seq database analysis (http://chip-atlas.org/, Supplementary Fig. S2B). The region displayed a significantly higher peak with gRNA-A or gRNA-B than the negative control gRNA, and it well matched the peak of the enhancer marker H3K27ac or DNase I hypersensitive site sequencing (DNase-seq), which is a marker for the open chromatin region [36] (Fig. 1b). To investigate whether this region would function as an enhancer, we generated five heterodeletion mutants of potential enhancer regions (Fig. 1c, d). All mutants showed decreased KLF5 expression, and the average (51.7\%) was significantly lower than that of parental cells (Fig. 1e, $P<0.01$ ), indicating that the identified region functions as an enhancer of KLF5 gene.

\section{KLF5 promoter-enhancer looping is organised by KLF5 protein and co-factors}

We hypothesised that the KLF5 protein might bind to the KLF5 promoter and/or the enhancer because ChIP-seq database showed this possibility in the KATO III gastric cancer cell line (http://chip-atlas.org/, Supplementary Fig. S3A). In addition, the JASPAR transcription-prediction tool [37] indicated that the transcription factor KLF5 protein is able to bind to both promoter and enhancer of KLF5 gene (Supplementary Fig. S3B, C). ChIP-qPCR analyses revealed that the KLF5 protein bound to the KLF5 promoter and enhancer in the HT29 and SW48 cell lines (Fig. 2a, b). In addition, we found that BRD4, MED1 (mediator complex protein) and RAD21 (cohesin complex protein) were also involved in the machinery constructing promoter-enhancer looping by binding to both regions (Fig. 2a, b). Moreover, a single knockdown of BRD4, MED1 and RAD21 and triple knockdown of these genes led to a modest decrease in KLF5 expression (Fig. 2c and Supplementary Fig. S3D). These results suggest that the KLF5 protein and co-factors may contribute to construct the promoter-enhancer looping of the KLF5 gene. Besides KLF5 protein, JASPAR transcription-prediction tool indicated that the transcription factors, Myeloid ecotropic viral integration site 1 (MEIS1), Reproductive homeobox on X-chromosome F1 (RHOXF1), Zinc finger protein 354C (ZNF354C) proteins might have the potential to bind to both promoter and enhancer of KLF5 gene (Supplementary Fig. S3B, C). Therefore, we performed ChIP-qPCR analyses and revealed that the MEIS1, RHOXF1 proteins, but not ZNF354C bound to both promoter and enhancer of KLF5 gene (Supplementary Fig. S3E).
The KLF5 enhancer is associated with cancer stem-like properties in CRC cells

In human clinical CRC tissues $(n=131)$ we found that the high KLF5 mRNA expression group had a worse prognosis than low KLF5 mRNA expression group when the cut-off point was set at a median value of $K L F 5$ mRNA expression ( $P=0.039$, Fig. 3a and Supplementary Table S2). To investigate the functional relevance of the KLF5 enhancer in cancer stem-like properties, we examined the chemoresistance, sphere-formation ability and expression level of CSC-related genes in heterodeletion mutants of the KLF5 enhancer. The heterodeletion mutants were more sensitive to 5-FU and oxaliplatin (L-OHP) and showed lower sphere-formation activity than parental cells (Fig. 3b, c and Supplementary Fig. S4A, $B)$. The cells had significantly decreased mRNA expression of the CSC-related markers BMI1, LGR5 and CD44v9 by RT-PCR (Fig. 3d), and the double-positive fractions of CD133 and CD44v9 in heterodeletion mutants also decreased to various extents compared with parental cells by flow cytometric analysis (parent $88.2 \%$ vs mutants 28.0, 61.0, 41.3, 28.7 and 69.7\%) (Fig. 3e and Supplementary Fig. S4C).

\section{The KLF5 promoter interacts with the CCAT1 TSS region located on a distinct chromosome}

We next analysed the interchromosomal interaction with the KLF5 promoter. Among many candidates, the most significant interaction was found with the CCAT1 TSS region located at chromosome 8 (Fig. 4a and Supplementary Fig. S5A). To clarify whether the CCAT1 TSS region functions as an enhancer of the KLF5 gene, we produced ten heterodeletion mutants of the CCAT1 TSS region (Fig. 4b-d). We found that these mutants clones displayed a large reduction in CCAT1 expression (Fig. 4e), but KLF5 expression levels were not affected (Fig. 4f). At the transcription level, knockdown of CCAT1 RNA did not affect KLF5 expression either (Fig. 4g).

We next investigated the possibility that the KLF5 gene product might conversely regulate CCAT1 expression and found that knockdown of KLF5 mRNA decreased CCAT1 expression in HT29 cells (Fig. 5a), and a KLF5 inhibitor, ML264, also suppressed CCAT1 expression in HT29 and SW48 cell lines (Fig. 5b). As a ChIP-seq database survey and a prediction of transcription factor-binding site showed that the KLF5 protein-binding region coincided with the CCAT1 TSS region (Supplementary Fig. S5B and C), our ChIP-qPCR analyses revealed that the KLF5 protein and BRD4, MED1 and RAD21 bound to the CCAT1 TSS region in the HT29 and SW48 cell lines (Fig. 5C). In heterodeletion mutants of the KLF5 enhancer, we found that CCAT1 expression markedly decreased, with an average of $21.8 \%$ (Fig. $5 d, P<0.01$ ). Moreover, BRD4, MED1 and RAD21 knockdown significantly decreased the CCAT1 expression (Supplementary Fig. S5D). These findings imply that the KLF5 protein and co-factors could participate also in the three-dimensional genome binding between KLF5 gene and CCAT1.

\section{KLF5 and CCAT1 expression are correlated in CRC clinical samples}

We next examined the expression of the KLF5 protein and CCAT1 RNA in 27 CRC tissue samples by immunohistochemistry and in situ hybridisation using RNA scope [32]. In normal tissue, nuclear KLF5 expression was observed predominantly at the colonic crypt bottom as previously reported [12] (Fig. 6a). In cancer tissues, KLF5 expression was observed in the nucleus or cytoplasm, and CRC cancer samples were divided into three groups based on the nuclear staining as follows: weak: $7.4 \%$, moderate: $37.0 \%$, and strong: $55.6 \%$ (Fig. $6 \mathrm{a}, \mathrm{b}$ ). On the other hand, CCAT1 was rarely expressed in normal epithelium and increased in cancer tissue (weak: $11.1 \%$, moderate: $40.7 \%$, strong: 48.2\%) (Fig. 6a, b). The KLF5 expression score was significantly correlated with that of CCAT1 $(P=0.0086$, Fig. 6b; scoring 


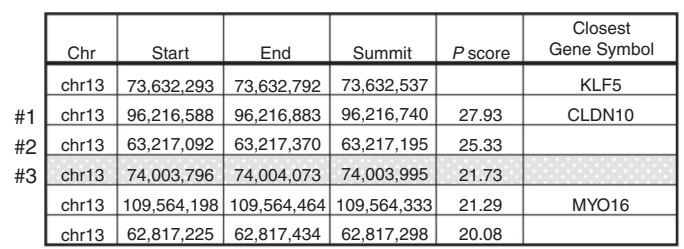

c
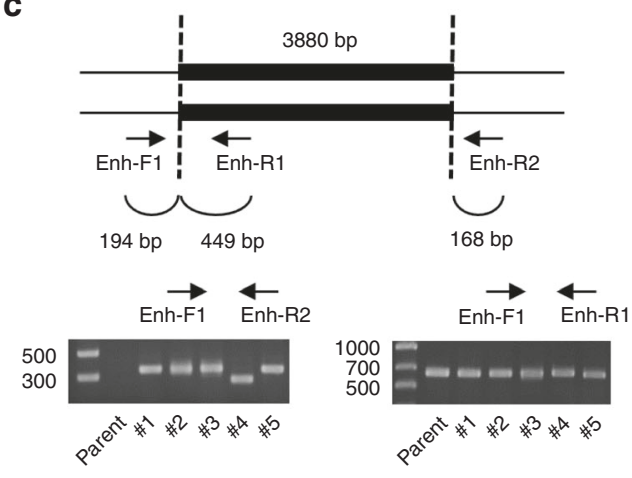

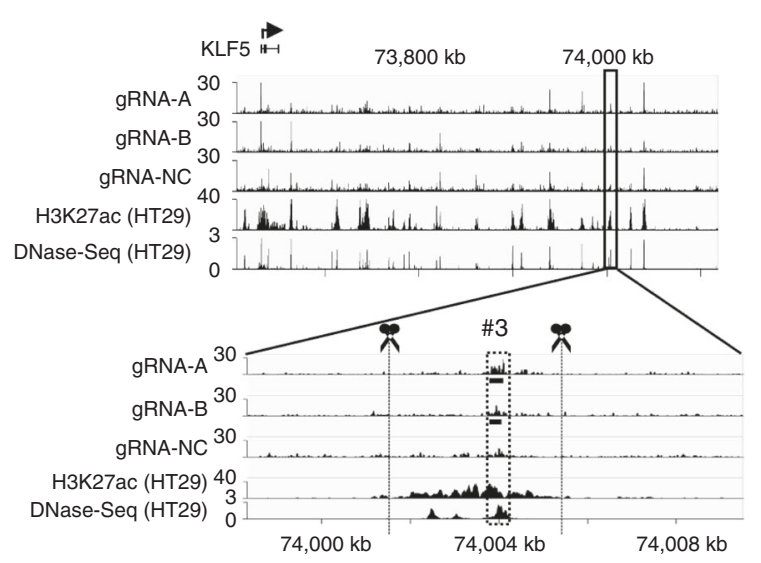

d

Chr13: $74,001,504-74,005,383$

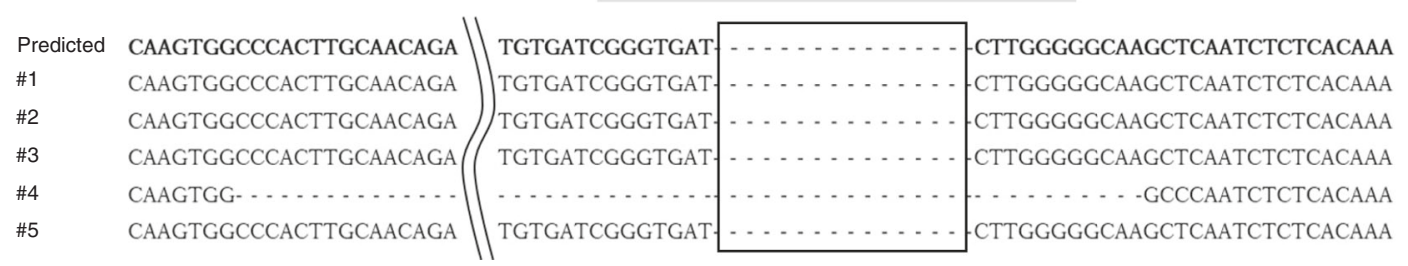

e

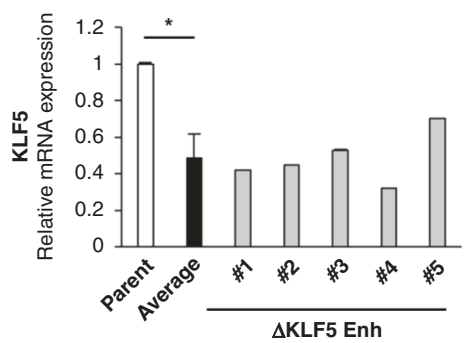

Fig. 1 Identification of the KLF5 enhancer region by in vitro enChIP-seq. a List of regions that bind to the KLF5 promoter by in vitro enChIPseq analysis. The regions of the top five highest $P$ scores on chromosome 13 are shown. First line shows the KLF5 promoter region including the target region of two gRNAs. The obtained reads from NGS were mapped to the human hg19 reference genome using the COBWeb algorithm. Peak calling was performed using the MACS peak detection algorithm at default settings on Strand NGS software version 3.4 (Agilent Technologies, Santa Clara, CA, USA). The peaks were determined using gRNA-A and gRNA-B as biological duplicates against gRNA-NC (negative control) filtered for $P$ score $(-\log 10(P$ values)) and fold change $\geq 2.0$. b Integrative genome viewer (IGV) tracks of in vitro enChIP-seq peaks (gRNA-A, gRNA-B, gRNA-NC) and ChIP-seq peaks of H3K27ac and DNase-seq peaks from the ChIP-Atlas database in HT29 cells. The region surrounded by a dotted square is the rank \#3rd peak listed in (a). Scissors indicate the position of the gRNAs to create the deletion mutants. c Schematic illustration of the KLF5 enhancer candidate region deleted by the CRISPR/Cas9 system. The positions of the primers for validation are indicated. All candidate clones of the deletion mutant were validated by PCR. Gel images show the PCR product amplified by the indicated primers (Enh-F1 and Enh-R2 or Enh-F1 and Enh-R1) in parental cells and five heterodeletion mutants. $\mathbf{d}$ Sequencing result of PCR products that are amplified by Enh-F1 and Enh-R2 primers in five heterodeletion mutants. The deleted region is surrounded by a square and the predicted sequence after deletion is shown as 'Predicted'. e Expression level of KLF5 mRNA in five deletion mutants. The relative value is calculated by the expression level of the parental cells. The average value of five heterodeletion mutants is also shown. ${ }^{*} P<0.01$.

system for KLF5 and CCAT1 is described in the legend of Fig. 6a). This correlation was also observed when we analysed in welldifferentiated adenocarcinoma samples $(P=0.0256$, Supplementary Fig. S6). In addition, qPCR analysis using RNA extracted from 131 CRC patients indicated that the expression levels of KLF5 mRNA and CCAT1 RNA were significantly correlated $(r=0.563, P<$ 0.0001) (Fig. 6C).

\section{DISCUSSION}

It is recently reported that $K L F 5$ transcripts are actively regulated by three-dimensional structural changes through promoter-enhancer binding in head and neck cancer cells [38]. We investigated this possibility in CRC because the enhancer markers H3K27ac and BRD4 showed similar peaks downstream of the KLF5 gene in HT29 colon cancer cells by a ChIP-seq database search (ChIP-Atlas: 
a

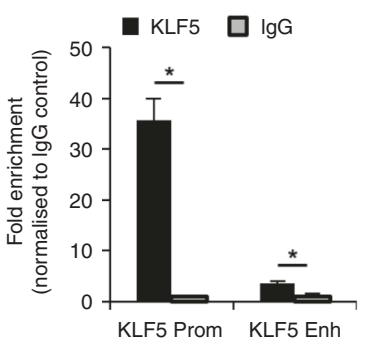

b

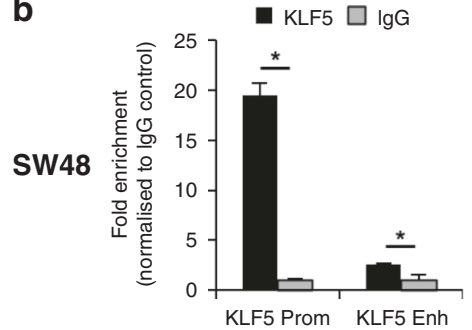

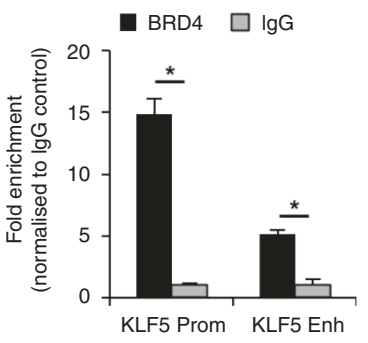
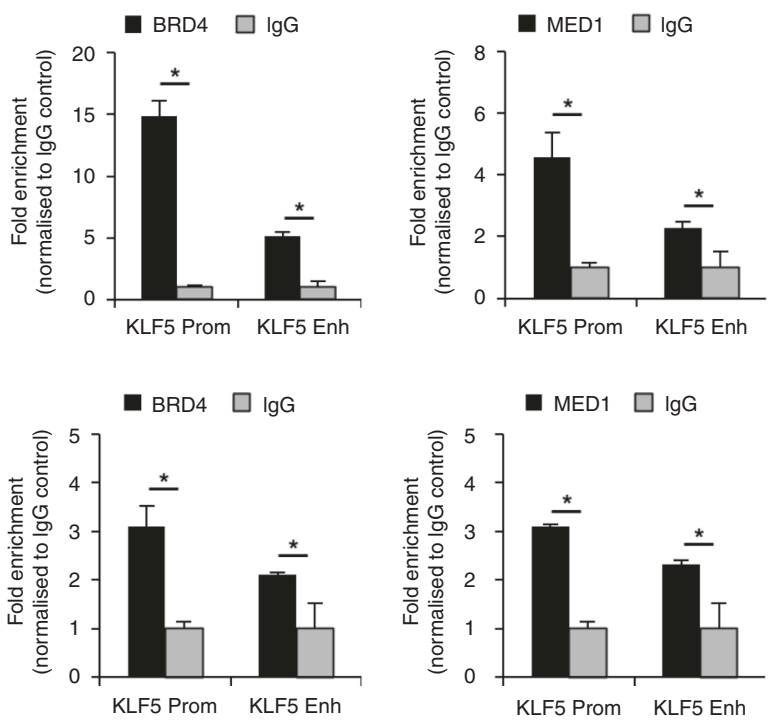
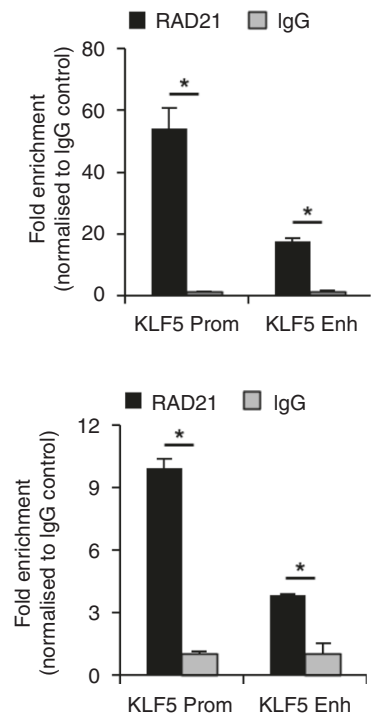

C
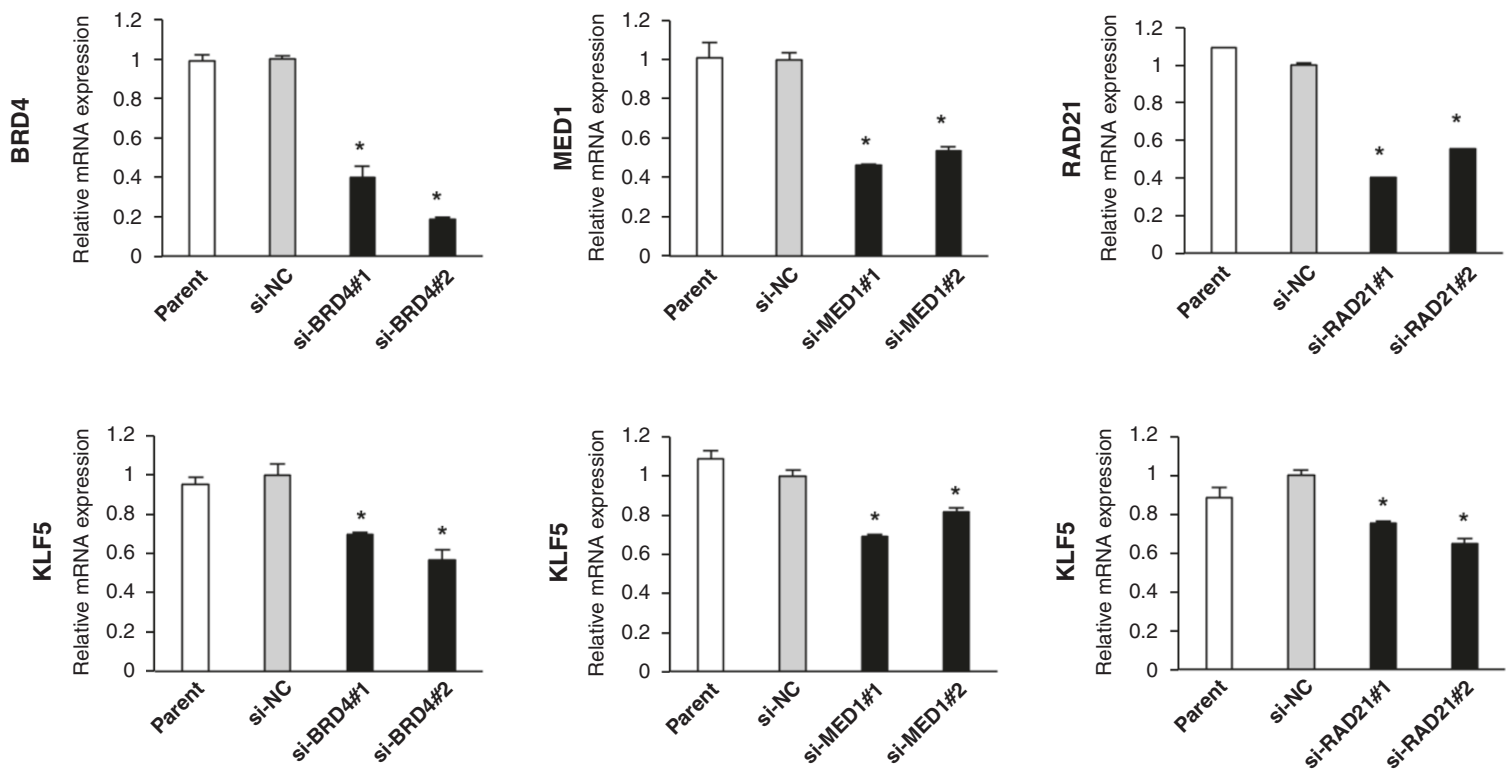

Fig. 2 KLF5 protein and co-factors bind to the KLF5 promoter and enhancer. $\mathbf{a}$, $\mathbf{b}$ The binding of the KLF5, BRD4, MED1 and RAD21 proteins to the KLF5 promoter and enhancer in HT29 (a) and SW48 (b) cell lines analysed by ChIP-qPCR. Fold enrichment normalised to the value of the lgG control is shown. ${ }^{*} P<0.01$. c Expression level of KLF5 mRNA in HT29 cells transfected with siRNAs against BRD4, MED1 and RAD21. The relative value is calculated by the expression level of the si-negative control transfected cells (siNC). ${ }^{*} P<0.01$.

https://chip-atlas.org/, Supplementary Fig. S7A). As result, we found that the KLF5 promoter bound not only to its enhancer region but also to IncRNA CCAT1.

Three-dimensional genome structure has been gradually uncovered due to the development of technologies such as $\mathrm{Hi}-\mathrm{C}$, chromatin interaction analysis by paired-end tag sequencing (ChIA-PET) analysis, and in vitro enChIP-seq method [20, 21, 39, 40]. Promoter- enhancer looping is constructed by the protein complex, including BRD4, the mediator complex, the cohesion complex and transcription factors $[41,42]$. Using the in vitro enChIP-seq method, we identified a tightly bound region $\sim 370 \mathrm{~kb}$ downstream of the KLF5 gene (chr13, $74,003,796-74,004,073,278 \mathrm{bp}$ ), and experiments using heterodeletion mutants of the enhancer verified that this region was indeed an enhancer of the KLF5 gene. Although this region partially overlapped with one of the enhancer regions reported in head and neck cancer (chr13, 74,002,153-74,004,229, 2077 bp) [38], we successfully encompassed the essential region. During the cloning process, we eventually obtained only five heterodeletion mutants and no homodeletion mutants out of 672 single cells into which the CRISPR-Cas9 system was transduced. One possible reason for missing the homodeletion clones is that the KLF5 gene is essential for cell survival. To support this idea, it was reported that KLF5 homozygous knockout mice died before embryonic day 8.5 [43], and even mice with conditional complete deletion of KLF5 in the gut died shortly after birth due to the absence of proliferation in the intestinal epithelium [44].

Cumulative evidence suggests that enhancer regions typically contain TF-binding motifs [36] For example, in murine embryonic stem cells, various enhancers are occupied by multiple TFs, including Oct4, Sox2 and Nanog [17]. Accordingly, we sought the TF-binding motif in the KLF5 promoter and enhancer by the JASPAR tool to further elucidate the molecular mechanism of KLF5 gene regulation. Notably, we found that the KLF5 protein itself was the candidate TF for regulating KLF5 expression and confirmed binding of the KLF5 protein to not only the enhancer region but also the promoter together with co-factors including BRD4, MED1 and RAD21 by ChIP-qPCR. A similar instance was 
a

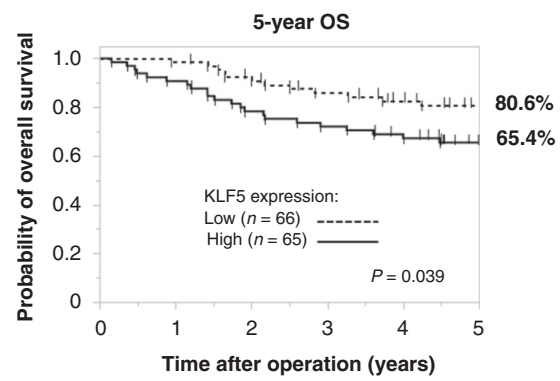

b
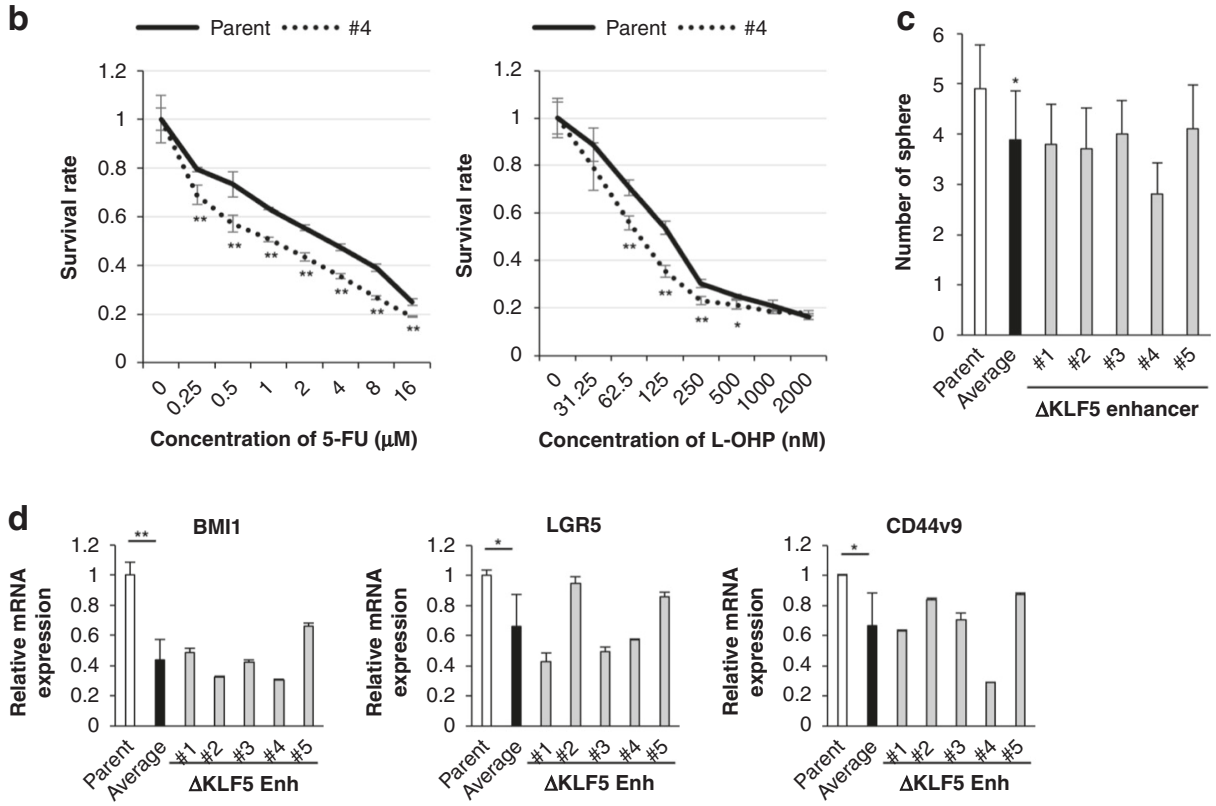

e

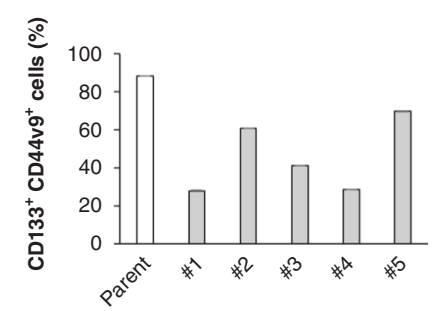

Fig. 3 The deletion mutants of the KLF5 enhancer show decreased cancer stem-like properties. a Kaplan-Meier survival analysis of overall survival for 131 CRC clinical samples classified by KLF5 expression score. The high KLF5 expression group had poorer survival when compared with the low KLF5 expression group $(P=0.039)$. When the cut-off point was set at a median value of KLF5 mRNA, there were no significant differences in patients' backgrounds between the high-KLF5 expression group $(n=65)$ and the low-KLF5 expression group ( $n=66$ ) (Supplementary Table S2). b The survival rate of parental cells and heterodeletion mutant clone \#4 of the KLF5 enhancer after 5-FU and L-OHP treatment. ${ }^{*} P<0.05,{ }^{* *} P<0.01$. c The number of spheres in parental cells and five heterodeletion mutants of the $K L F 5$ enhancer. Five hundred cells were seeded, and the number was counted 3 weeks after seeding. The spheres larger than $50 \mu \mathrm{m}$ were counted. The average number of five heterodeletion mutants is also shown. ${ }^{*} P<0.01$. d The expression level of CSC-related genes in parental cells and five heterodeletion mutants of the KLF5 enhancer. The relative value is calculated by the expression level of the parental cells. The average value of five heterodeletion mutants is also shown. ${ }^{*} P<0.05,{ }^{*} P<0.01$. e The expression levels of CD44v9 and CD 133 were analysed by flow cytometry in parental cells and five heterodeletion mutants of the KLF5 enhancer. The percentage of double-positive cells are shown.

recently reported in oesophageal squamous cell carcinoma cell lines; KLF5 participated in the core-regulatory circuitry (together with TFs, TP63 and SOX2 and co-factors) to construct the threedimensional genome of the $A L D H 3 A 1$ or EGFR gene [45]. Our finding may be more unique regarding autoactivation because the regulatory circuitry containing the KLF5 protein contributes to the three-dimensional genome structure of the KLF5 gene. On the other hand, we found that other two transcription factors, MEIS1 and RHOXF1, also bound to both KLF5 promoter and enhancer region, despite the binding may be weaker compared to the KLF5 protein. MEIS1 belongs to a family of the three amino acid loop extension (TALE) homeodomain transcription factor and it was reported that MEIS1 functions as the regulator of the cell cycle, cell proliferation and differentiation [46]. Of note, it was reported that MEIS1 is involved in superenhancer associated gene expression in combination with EWS-FLI in Ewing sarcoma [47]. RHOXF1 (originally called as OTEX and hPEPP1) is a member of Rhox gene family, which is expressed in ovary, testis, epididymis, prostate and mammary gland [48] and malignant diseases of prostate cancer, leukaemia and CRC $[49,50]$. Further studies will be needed on whether these proteins are also involved in the core-regulatory circuitry. 
a

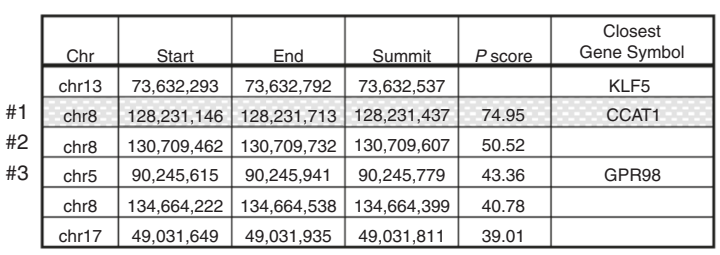

C

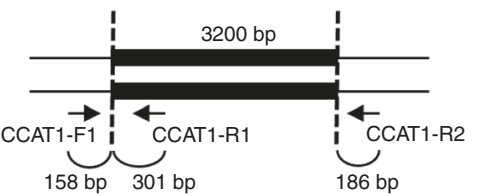

CCAT1-F1 $\leftarrow$ CCAT1-R2
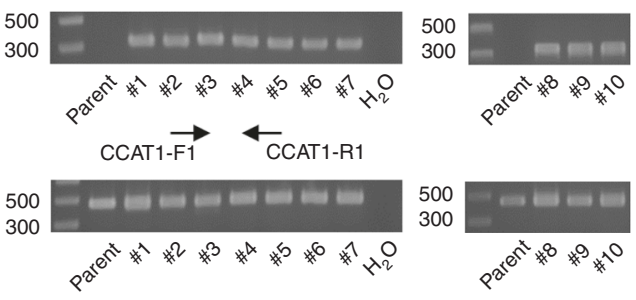

e

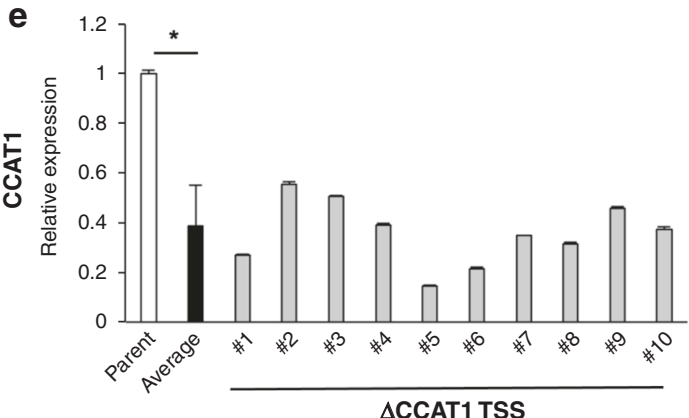

b

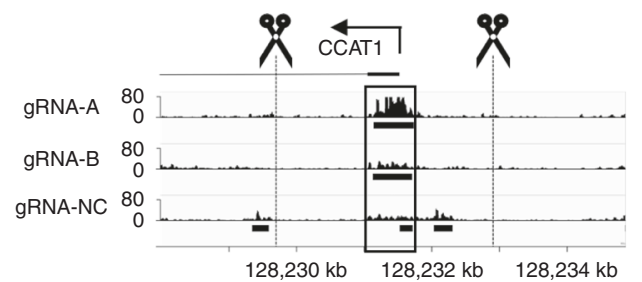

d

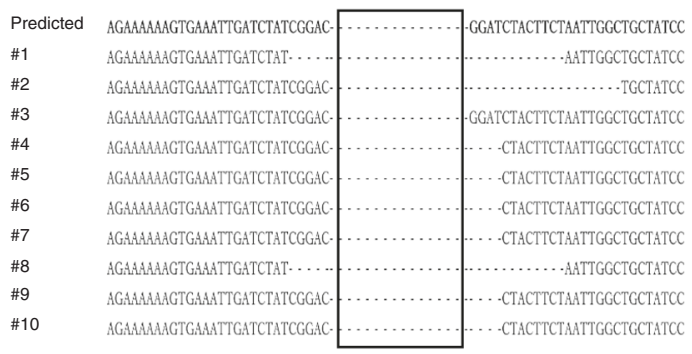

f

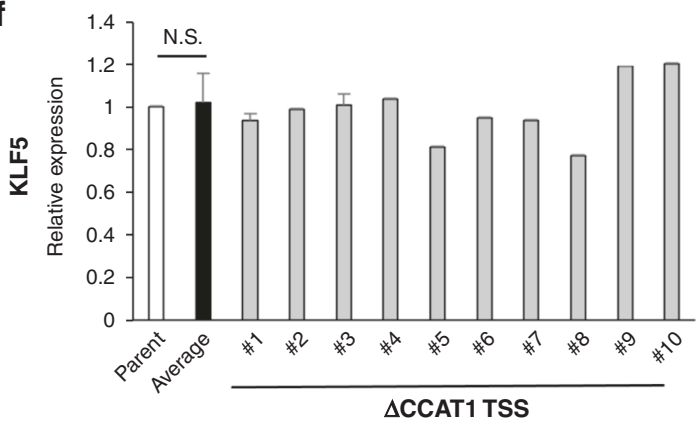

g

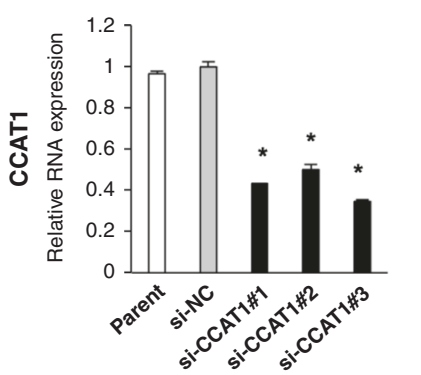

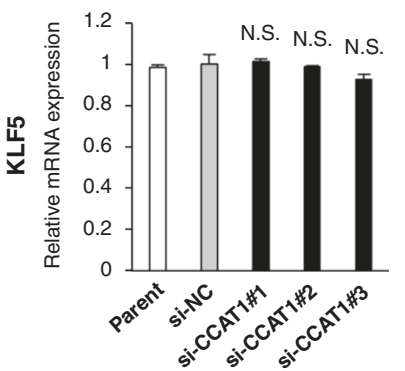

Fig. 4 The KLF5 promoter binds to the CCAT1 TSS region. a List of regions that bind to the KLF5 promoter by analysing the in vitro enChIPseq data. The regions of the top five highest $P$ scores in all chromosomes are shown. First line shows the KLF5 promoter region including the target region of two gRNAs. b IGV track of the CCAT1 TSS region. Scissors indicate the position of the gRNAs for creating the deletion mutants. The region surrounded by a square is the \#1st peak. c Schematic illustration of the CCAT1 TSS region deleted by the CRISPR/ Cas9 system. The positions of the primers for validation are indicated. All candidate clones of the deletion mutant were validated by PCR. Gel images show the PCR product amplified by the indicated primers (CCAT1-F1 and CCAT1-R2 or CCAT1-F1 and CCAT1-R1) in parental cells and ten heterodeletion mutants. d Sequencing results of PCR products amplified by CCAT1-F1 and CCAT1-R2 primers in ten heterodeletion mutants. The deleted region is indicated as a square and the predicted sequence after deletion is shown as 'Predicted'. e Expression level of CCAT1 RNA in ten heterodeletion mutants of the CCAT1 TSS region. The relative value is calculated by the expression level of the parental cells. The average value of ten heterodeletion mutants is also shown. ${ }^{*} P<0.01$. $\mathbf{f}$ Expression level of KLF5 mRNA in ten heterodeletion mutants of the CCAT1 TSS region. The relative value is calculated by the expression level of the parental cells. The average value of ten heterodeletion mutants is also shown. N.S. not significant. g Expression levels of CCAT1 RNA and KLF5 mRNA in CCAT1 knockdown cells. The relative value is calculated by the expression level of the si-negative control transfected cells (siNC). ${ }^{*} P<0.01$. N.S. not significant. 
a

HT29

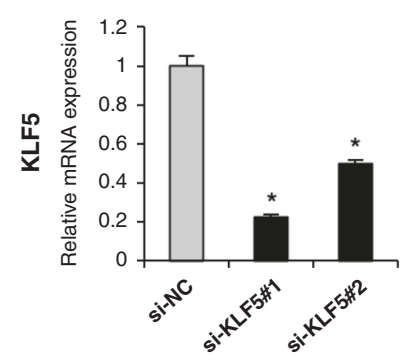

b

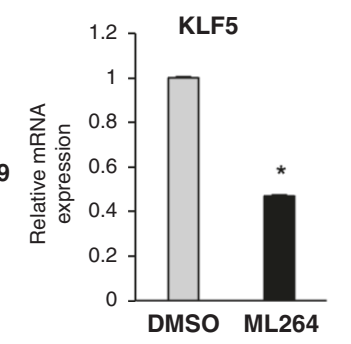

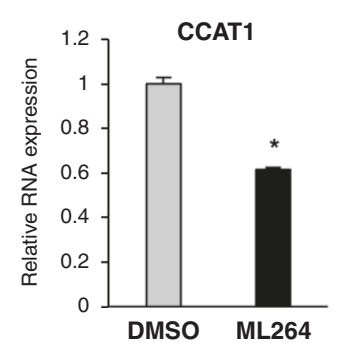

C
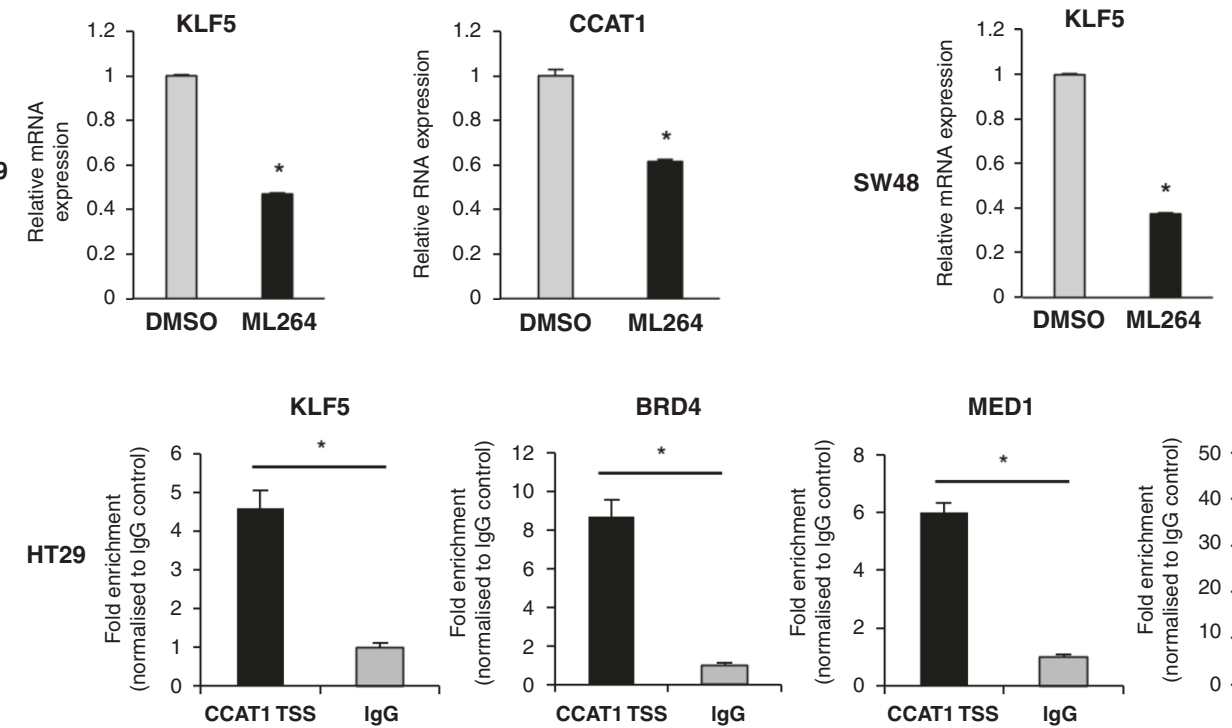

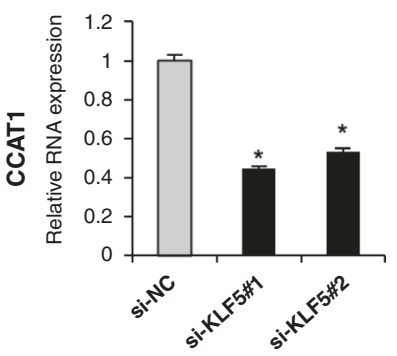

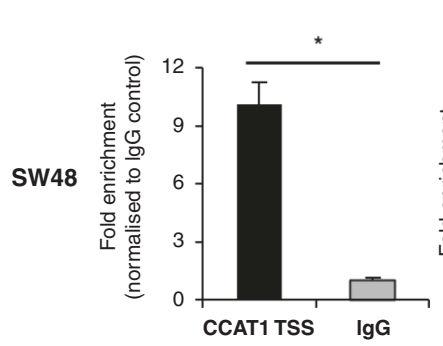
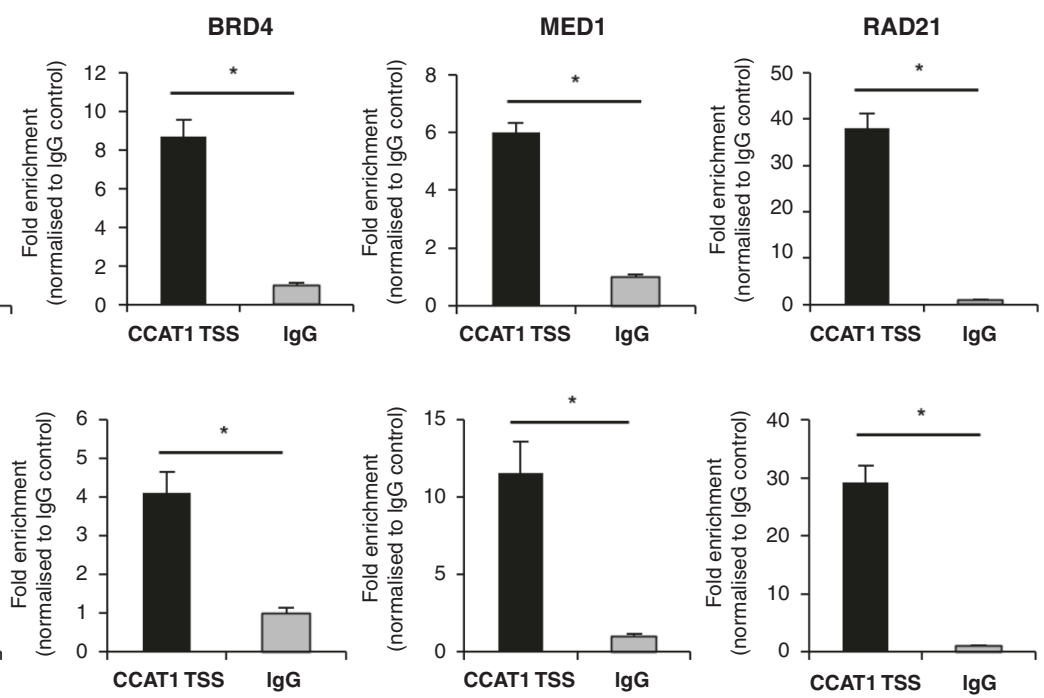

d

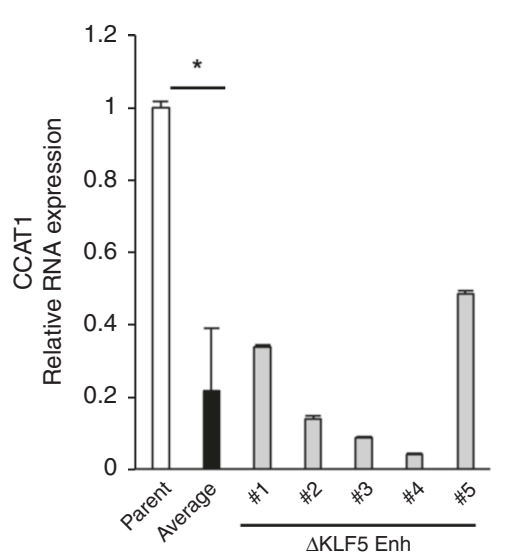

Fig. 5 KLF5 protein regulates CCAT1 expression. a Expression levels of CCAT1 RNA and KLF5 mRNA in KLF5 knockdown cells. The relative value is calculated by the expression level of the si-negative control transfected cells (siNC). ${ }^{*} P<0.01$. $\mathbf{b}$ Expression levels of $K L F 5$ mRNA and CCAT1 RNA in ML264-treated cells (HT29, SW48). ML264 (10 $\mu \mathrm{M})$ was treated for $24 \mathrm{~h}$. The relative value is calculated by the expression level of DMSO-treated control cells. ${ }^{*} P<0.01$. c The binding of the KLF5, BRD4, MED1 and RAD2 1 proteins to the CCAT1 TSS region in the HT29 (top) and SW48 (bottom) cell lines analysed by ChIP-qPCR. The fold enrichment normalised to the value of the IgG control is shown. ${ }^{*} P<0.01$. d Expression level of CCAT1 RNA in five heterodeletion mutants of the KLF5 enhancer. The relative value is calculated by the expression level of the parental cells. The average value of five heterodeletion mutants is also shown. ${ }^{*} P<0.01$. 
a

Cancer

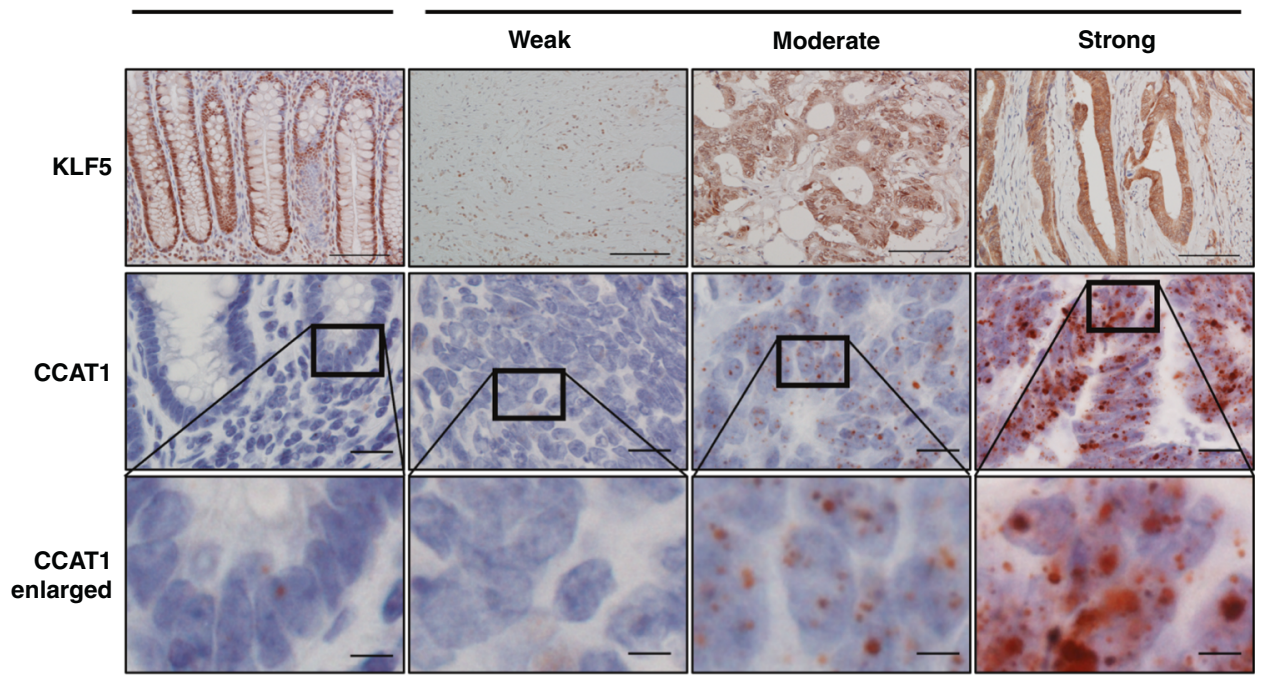

b

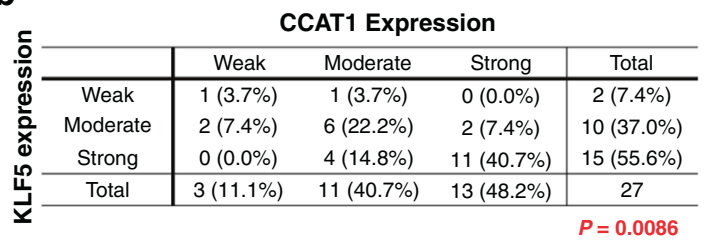

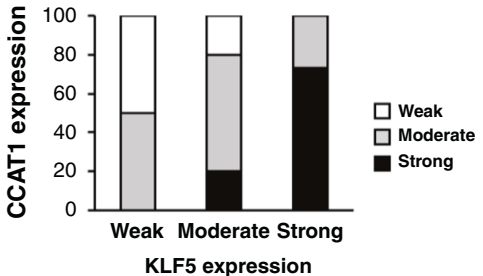

C

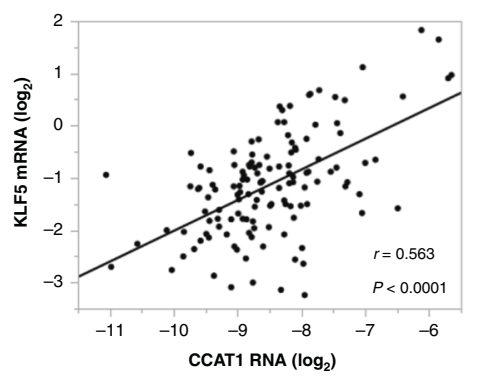

d

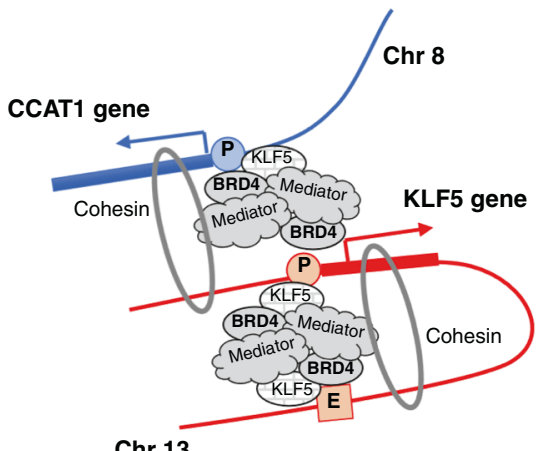

Fig. 6 KLF5 and CCAT1 expression is correlated in CRC tissues. a Representative images of KLF5 protein and CCAT1 RNA expression in normal colorectal epithelium and CRC. The expression level of KLF5 in tumour cells was evaluated at $\times 100$ magnification. Nuclear staining of the KLF5 protein was scored based on the percentage of positive cells as follows: weak: $0-10 \%$, moderate: $11-50 \%$, strong: $51-100 \%$. The expression level of CCAT1 in tumour cells was evaluated at $\times 100$ magnification and scored as previously reported [32]. Score 0 was defined as weak, score 1-2 was defined as moderate, and score 3-4 was defined as strong. The bottom panels show the higher magnification image of the framed area. Scale bar: $100 \mu \mathrm{m}$ (top), $20 \mu \mathrm{m}$ (middle), $5 \mu \mathrm{m}$ (bottom). b The percentage of each score (weak, moderate, strong) of KLF5 protein and CCAT1 RNA expression in CRC clinical samples. The bar chart is also shown. c The correlation of KLF5 mRNA and CCAT1 RNA expression in 131 CRC clinical samples. The expression levels of $K L F 5$ mRNA and CCAT1 RNA were significantly correlated ( $r=0.563, P<0.0001)$. d Illustration model showing the gene regulation mechanism for KLF5 and CCAT1 via the three-dimensional genome structure.

Long-range genome interactions, as well as local genome interactions, are known to regulate cell-type-specific gene expression and maintain cell identity. Interchromosomal interactions have been demonstrated between SOX9 and the IncRNA CISTR-ACT gene or the ATF4 and FIRRE genes to serve biological processes including mammalian development and differentiation, as well as cancer stemness [51]. For the interchromosomal interaction, we focused on the specific binding between the KLF5 promoter and the CCAT1 TSS region because the peak of this region was even higher and more significant than that of intrachromasomal interaction including KLF5 promoter-enhancer interaction (Figs. 1a, b and 4a, b).
Studies have shown that the CCAT1 genomic region is a part of the superenhancer for the MYC gene and that CCAT1 RNA facilitates EGFR expression through activation of the EGFR enhancer by recruiting the transcription factors p63 and SOX2 [52]. However, in the current case, the KLF5 expression level was not altered when CCAT1 DNA was heterogeneously deleted or the CCAT1 transcript was suppressed by the specific siRNAs. Conversely, KLF5 knockdown by specific siRNAs and treatment with the KLF5 inhibitor suppressed CCAT1 expression. Since the KLF5 protein bound to the CCAT1 TSS region as well as the KLF5 promoter and enhancer together with co-factors (BRD4, MED1, RAD21), we speculate that the KLF5 gene product participates in 
the core-regulatory circuitry and may regulate CCAT1 expression. We performed the knockdown experiment by treating the single or triple combination of siRNAs against co-factors (BRD4, mediator, cohesin) and the result showed that knockdown efficiency was sufficient in both conditions but the downregulation of KLF5 was still modest. These results suggest that knockdown of co-factors alone was not enough to achieve the complete disruption of the regulatory machinery for KLF5 gene expression although it is indeed partially involved. It is of interest, according to the CCLE gene expression database (https://portals.broadinstitute.org/ccle), that both CCAT1 and KLF5 RNAs are upregulated in gastrointestinal cancers and downregulated in leukaemia and lymphoma (Supplementary Fig. S7B, C). In clinical samples, we verified a tight correlation between the expression of KLF5 and CCAT1. These findings imply the coordinated expression of the two genes in CRC and possibly in other type of human cancers.

The KLF5 enhancer region plays a biologically important role in cancer stem-like properties. Thus, even heterogenous deletion mutant clones exhibited a decrease in CSC markers such as LGR5, $B M 11, C D 133$, and CD44v9, restored susceptibility to chemotherapy, and reduced sphere formation. This could be attributed to the downregulation of KLF5 because the KLF5 inhibitor suppressed CSC-related gene expression in CRC cell lines (Supplementary Fig. S8A, B). Moreover, we and another group recently showed that the treatment of miR-4711-5p targeting to the 3'-UTR of KLF5 mRNA or KLF5-siRNA attenuated the CSC properties in CRC cell lines [10,11]. We also showed in this study that high KLF5 expression in CRC clinical samples was correlated with poor prognosis, which is consistent with other studies [10]. In addition, studies reported that CCAT1 stimulated symmetric division and self-renewal, which are hallmarks of CSCs in lung cancer, and that CCAT1 is required for the maintenance of stemness, proliferation, migration and invasion of breast cancer stem cells $[53,54]$. It was also reported that $B M I 1$, which is one of the CSC-related genes, was decreased by the knockdown of CCAT1 [55]. Because CCAT1 expression was decreased in the deletion mutants of the KLF5 enhancer, it is suggested that the KLF5 enhancer may play a crucial role in the maintenance of cancer stemness by regulating KLF5 and CCAT1. Taken together, these results show that targeting therapeutics against the KLF5 enhancer, e.g., blockade of KLF5 promoter-enhancer binding by the decoy oligonucleotide strategy may be an efficient therapeutic option for CSCs.

In summary, we identified an enhancer of KLF5 downstream of the KLF5 gene that interacted with the KLF5 promoter, and this enhancer region was associated with CSC properties in CRC. We also found that the KLF5 promoter interacted with the CCAT1 TSS region, which is located on different chromosomes. Notably, our results suggest that the core-regulatory circuitry containing the KLF5 gene product and co-factors help to construct threedimensional genome interaction and regulates the gene expression of KLF5 and CCAT1 (Fig. 6d), and this mechanism may facilitate the maintenance of CSC properties in CRC.

\section{Reporting summary}

Further information on research design is available in the Nature Research Reporting Summary linked to this article.

\section{DATA AVAILABILITY}

The ChIP-Atlas (http://chip-atlas.org/) database was used for the analysis of ChIP-seq and DNase-seq data. The following datasets were analysed in this study: GEO ID: GSM1890734, GSM2400470, GSM1890736 and GSM1250899. For the analysis of mRNA expression in the multiple types of cancer cell lines, the CCLE (The Cancer Cell Line Encyclopedia) database (https://portals.broadinstitute.org/ccle) was used. The accession number of the in vitro enChIP-seq data in this study is DRA007368.

\section{REFERENCES}

1. Bialkowska AB, Yang VW, Mallipattu SK. Krüppel-like factors in mammalian stem cells and development. Development. 2017;144:737-54.

2. Ema M, Mori D, Niwa H, Hasegawa Y, Yamanaka Y, Hitoshi S, et al. Krüppel-like factor 5 is essential for blastocyst development and the normal self-renewal of mouse ESCs. Cell Stem Cell. 2008;3:555-67.

3. Kenchegowda D, Swamynathan S, Gupta D, Wan H, Whitsett J, Swamynathan SK. Conditional disruption of mouse KIf5 results in defective eyelids with malformed meibomian glands, abnormal cornea and loss of conjunctival goblet cells. Dev Biol. 2011;356:5-18.

4. Wan $H$, Luo F, Wert SE, Zhang L, Xu Y, Ikegami M, et al. Kruppel-like factor 5 is required for perinatal lung morphogenesis and function. Development. 2008; 135:2563-72.

5. Bell SM, Zhang L, Mendell A, Xu Y, Haitchi HM, Lessard JL, et al. Kruppel-like factor 5 is required for formation and differentiation of the bladder urothelium. Dev Biol. 2011;358:79-90.

6. Kim CK, Saxena M, Maharjan K, Song JJ, Shroyer KR, Bialkowska AB, et al. Krüppellike factor 5 regulates stemness, lineage specification, and regeneration of intestinal epithelial stem cells. Cell Mol Gastroenterol Hepatol. 2020;9:587-609.

7. Nandan MO, Ghaleb AM, Bialkowska AB, Yang VW. Krüppel-like factor 5 is essential for proliferation and survival of mouse intestinal epithelial stem cells. Stem Cell Res. 2015;14:10-19.

8. David CJ, Huang YH, Chen M, Su J, Zou Y, Bardeesy N, et al. TGF- $\beta$ tumor suppression through a lethal EMT. Cell. 2016;164:1015-30.

9. Clevers $\mathrm{H}$. The cancer stem cell: premises, promises and challenges. Nat Med. 2011;17:313-9.

10. Takagi Y, Sakai N, Yoshitomi H, Furukawa K, Takayashiki T, Kuboki S, et al. High expression of Krüppel-like factor 5 is associated with poor prognosis in patients with colorectal cancer. Cancer Sci. 2020;111:2078-92.

11. Morimoto $\mathrm{Y}$, Mizushima $\mathrm{T}, \mathrm{Wu} \mathrm{X}$, Okuzaki $\mathrm{D}$, Yokoyama $\mathrm{Y}$, Inoue $\mathrm{A}$, et al. miR4711-5p regulates cancer stemness and cell cycle progression via KLF5, MDM2 and TFDP1 in colon cancer cells. Br J Cancer. 2020;122:1-13.

12. Nakaya T, Ogawa S, Manabe I, Tanaka M, Sanada M, Sato T, et al. KLF5 regulates the integrity and oncogenicity of intestinal stem cells. Cancer Res. 2014;74:2882-91.

13. Marsman J, Horsfield JA. Long distance relationships: Enhancer-promoter communication and dynamic gene transcription. Biochim Biophys Acta - Gene Regul Mech. 2012;1819:1217-27.

14. Zheng $\mathrm{H}, \mathrm{Xie} W$. The role of 3D genome organization in development and cell differentiation. Nat Rev Mol Cell Biol. 2019;20:535-50.

15. Wilber A, Nienhuis AW, Persons DA. Transcriptional regulation of fetal to adult hemoglobin switching: new therapeutic opportunities. Blood. 2011;117:3945-53.

16. Yokoyama Y, Zhu H, Lee JH, Kossenkov AV, Wu SY, Wickramasinghe JM, et al. BET inhibitors suppress ALDH activity by targeting ALDH1A1 super-enhancer in ovarian cancer. Cancer Res. 2016;76:6320-30.

17. Hnisz D, Abraham BJ, Lee TI, Lau A, Saint-André V, Sigova AA, et al. Superenhancers in the control of cell identity and disease. Cell. 2013;155:934-47.

18. Borggrefe $T$, Yue X. Interactions between subunits of the mediator complex with gene-specific transcription factors. Semin Cell Dev Biol. 2011;22:759-68.

19. Perea-Resa C, Wattendorf L, Marzouk S, Blower MD. Cohesin: behind dynamic genome topology and gene expression reprogramming. Trends Cell Biol. 2021; 31:760-73.

20. Fujita T, Yuno M, Fujii H. Efficient sequence-specific isolation of DNA fragments and chromatin by in vitro enChIP technology using recombinant CRISPR ribonucleoproteins. Genes Cells. 2016;21:370-7.

21. Fujita T, Kitaura F, Yuno M, Suzuki $Y$, Sugano S, Fujii H. Locus-specific ChIP combined with NGS analysis reveals genomic regulatory regions that physically interact with the Pax5 promoter in a chicken B cell line. DNA Res. 2017;24:537-48.

22. Nissan A, Stojadinovic A, Mitrani-Rosenbaum S, Halle D, Grinbaum R, Roistacher $M$, et al. Colon cancer associated transcript-1: A novel RNA expressed in malignant and pre-malignant human tissues. Int J Cancer. 2012;130:1598-606.

23. McCleland ML, Mesh K, Lorenzana E, Chopra VS, Segal E, Watanabe C, et al. CCAT1 is an enhancer-templated RNA that predicts BET sensitivity in colorectal cancer. J Clin Investig. 2016;126:639-52.

24. Xin Y, Li Z, Shen J, Chan MTV, Wu WKK. CCAT1: a pivotal oncogenic long noncoding RNA in human cancers. Cell Prolif. 2016;49:255-60.

25. Mizrahi I, Mazeh H, Grinbaum R, Beglaibter N, Wilschanski M, Pavlov V, et al. Colon cancer associated transcript-1 (CCAT1) expression in adenocarcinoma of the stomach. J Cancer. 2015;6:105-10.

26. Zhang E, Han L, Yin D, He X, Hong L, Si X, et al. H3K27 acetylation activated-long non-coding RNA CCAT1 affects cell proliferation and migration by regulating SPRY4 and HOXB13 expression in esophageal squamous cell carcinoma. Nucleic Acids Res. 2017;45:3086-101.

27. Ghafouri-Fard S, Taheri M. Colon cancer-associated transcripts 1 and 2: roles and functions in human cancers. J Cell Physiol. 2019;234:14581-14600. 
28. He X, Tan X, Wang X, Jin H, Liu L, Ma L, et al. C-Myc-activated long noncoding RNA CCAT1 promotes colon cancer cell proliferation and invasion. Tumour Biol. 2014;35:12181-8.

29. Yang C, Pan Y, Deng SP. Downregulation of IncRNA CCAT1 enhances 5-fluorouracil sensitivity in human colon cancer cells. BMC Mol Cell Biol. 2019; 20:1-11.

30. Bialkowska A, Crisp M, Madoux F, Spicer T, Knapinska A, Mercer B, et al. ML264: an antitumor agent that potently and selectively inhibits Krüppel-like factor five (KLF5) expression: a probe for studying colon cancer development and progression. Probe Reports from the NIH Molecular Libraries Program. Bethesda, MD; 2010.

31. Inoue A, Yamamoto $H$, Uemura $M$, Nishimura J, Hata T, Takemasa $I$, et al. MicroRNA-29b is a novel prognostic marker in colorectal cancer. Ann Surg Oncol. 2015;22:S1410-8.

32. Wang $H$, Su N, Wang LC, Wu X, Bui S, Nielsen A, et al. Quantitative ultrasensitive bright-field RNA in situ hybridization with RNAscope. In: Nielsen BS, editor. In situ hybridization protocols. New York, NY: Springer New York; p 201-12, 2014.

33. Lee TI, Johnstone SE, Young RA. Chromatin immunoprecipitation and microarraybased analysis of protein location. Nat Protoc. 2006;1:729-48.

34. Mali P, Yang L, Esvelt KM, Aach J, Guell M, DiCarlo JE, et al. RNA-guided human genome engineering via Cas9. Science. 2013;339:823-6.

35. Fujita $T$, Yuno $M$, Fujii $H$. Allele-specific locus binding and genome editing by CRISPR at the p16INK4a locus. Sci Rep. 2016;6:30485.

36. Shlyueva D, Stampfel G, Stark A. Transcriptional enhancers: from properties to genome-wide predictions. Nat Rev Genet. 2014;15:272-86.

37. Khan A, Fornes O, Stigliani A, Gheorghe M, Castro-Mondragon JA, Van Der Lee $R$ et al. JASPAR 2018: update of the open-access database of transcription factor binding profiles and its web framework. Nucleic Acids Res. 2018;46:D260-D266.

38. Zhang X, Choi PS, Francis JM, Gao GF, Campbell JD, Ramachandran A, et al. Somatic superenhancer duplications and hotspot mutations lead to oncogenic activation of the KLF5 transcription factor. Cancer Discov. 2018;8:108-25.

39. Lieberman-Aiden $E$, van Berkum NL, Williams $L$, Imakaev M, Ragoczy $T$, Telling A, et al. Comprehensive mapping of long-range interactions reveals folding principles of the human genome. Science. 2009;326:289-93.

40. Fullwood MJ, Liu MH, Pan YF, Liu J, Xu H, Mohamed YB, et al. An oestrogenreceptor-alpha-bound human chromatin interactome. Nature. 2009;462:58-64.

41. Sengupta S, George RE. Super-enhancer-driven transcriptional dependencies in cancer. Trends Cancer 2017;3:269-81.

42. Stadhouders R, Filion GJ, Graf T. Transcription factors and 3D genome conformation in cell-fate decisions. Nature. 2019;569:345-54.

43. Arvidsson A, Collin T, Kirk D, Kokaia Z, Lindvall O. Neuronal replacement from endogenous precursors in the adult. Nat Med. 2003;9:548-53.

44. McConnell BB, Kim SS, Yu K, Ghaleb AM, Takeda N, Manabe I, et al. Krppel-like factor 5 is important for maintenance of crypt architecture and barrier function in mouse intestine. Gastroenterology. 2011;141:1302-13.

45. Jiang $Y$, Jiang $Y$, Li C, Zhang $Y$, Dakle $P$, Kaur $H$, et al. TP63, SOX2, and KLF5 establish a core regulatory circuitry that controls epigenetic and transcription patterns in esophageal squamous cell carcinoma cell lines. Gastroenterology. 2020;159:1311-27.

46. Jiang $M, X u S$, Bai $M$, Zhang $A$. The emerging role of MEIS1 in cell proliferation and differentiation. Am J Physiol Cell Physiol. 2021;320:C264-C269.

47. Lin L, Huang $M$, Shi $X$, Mayakonda A, Hu K, Jiang $Y$, et al. Super-enhancerassociated MEIS1 promotes transcriptional dysregulation in Ewing sarcoma in cooperation with EWS-FLI1. Nucleic Acids Res. 2019;47:1255-67.

48. Geserick C, Weiss B, Schleuning W, Haendler B. OTEX, an androgen-regulated human member of the paired-like class of homeobox genes. Biochem J. 2002; 375:367-75.

49. Wayne CM, Maclean JA, Cornwall G, Wilkinson MF. Two novel human X-linked homeobox genes, hPEPP1 and hPEPP2, selectively expressed in the testis. Gene. 2002;301:1-11.

50. Li Q, O'Malley ME, Bartlett DL, Guo ZS. Homeobox gene Rhox5 is regulated by epigenetic mechanisms in cancer and stem cells and promotes cancer growth Mol Cancer. 2011;10:63.

51. Maass PG, Barutcu AR, Rinn JL. Interchromosomal interactions: a genomic love story of kissing chromosomes. J Cell Biol. 2019;218:27-38.

52. Jiang $Y$, Jiang $Y Y$, Xie JJ, Mayakonda $A$, Hazawa $M$, Chen $L$, et al. Co-activation of super-enhancer-driven CCAT1 by TP63 and SOX2 promotes squamous cancer progression. Nat Commun. 2018;9:3619.

53. Tang $T$, Guo $C$, Xia T, Zhang R, Zen $K$, Pan $Y$, et al. LncCCAT1 promotes breast cancer stem cell function through activating WNT/ $\beta$-catenin signaling. Theranostics. 2019:9:7384-402.

54. Xu C, Xiao G, Zhang B, Wang M, Wang J, Liu D, et al. CCAT1 stimulation of the symmetric division of NSCLC stem cells through activation of the Wnt signalling cascade. Gene Ther. 2018;25:4-12.
55. Li N, Jiang K, Fang LP, Yao LL, Yu Z. Knockdown of long noncoding RNA CCAT1 inhibits cell growth, invasion and peritoneal metastasis via downregulation of Bmi-1 in gastric cancer. Neoplasma. 2018;65:736-44.

\section{ACKNOWLEDGEMENTS}

We thank Wu X, Kitagawa K, Sugiyama M, Matsui M, Kago N, Nakayama C, Umekawa I (Department of Molecular Pathology, Division of Health Sciences, Graduate School of Medicine, Osaka University) for technical assistance. We thank the laboratory member of the Department of Computational Biology and Medical Sciences, Graduate School of Frontier Sciences, the University of Tokyo for NGS analysis. We also thank Dr. George Church for providing the hCas9 plasmid (Addgene \#41815).

\section{AUTHOR CONTRIBUTIONS}

Conception and design: YY, HU, MM and HY. Development of methodology: YY, KA, TF and HF. Acquisition of the data: TT, HT, DO, KA, HI and MU. Analysis and interpretation of the data (e.g., statistical analysis, biostatistics and computational analysis): $Y Y, D O, N M$ SK, HE and HY. Writing the manuscript: $\Pi, Y Y$ and HY. Administrative, technical or material support: TF, HU and HF. Study supervision: YY, HU, MM, YD and HY.

\section{FUNDING INFORMATION}

This work was supported by JSPS KAKENHI Grant Number $18 \mathrm{~K} 16360$ (to TT), JSPS KAKENHI Grant Number 16H06279 (PAGS), 17K15618, 19K16541, The Osaka Cancer Foundation Award and Takeda Science Foundation (to YY) and a grant from the Kagoshima Shinsangyo Sousei Investment Limited Partnership (its general partner is Kagoshima Development Co., Ltd).

\section{COMPETING INTERESTS}

The authors declare no competing interests.

\section{ETHICS APPROVAL AND CONSENT TO PARTICIPATE}

This study was approved by the Ethics Committee of Osaka University Hospital (No. 15144).

\section{CONSENT TO PUBLISH}

Informed consent signatures were obtained from all patients.

\section{ADDITIONAL INFORMATION}

Supplementary information The online version contains supplementary material available at https://doi.org/10.1038/s41416-021-01579-4.

Correspondence and requests for materials should be addressed to Yuhki Yokoyama.

Reprints and permission information is available at http://www.nature.com/ reprints

Publisher's note Springer Nature remains neutral with regard to jurisdictional claims in published maps and institutional affiliations.

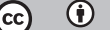

Open Access This article is licensed under a Creative Commons Attribution 4.0 International License, which permits use, sharing, adaptation, distribution and reproduction in any medium or format, as long as you give appropriate credit to the original author(s) and the source, provide a link to the Creative Commons license, and indicate if changes were made. The images or other third party material in this article are included in the article's Creative Commons license, unless indicated otherwise in a credit line to the material. If material is not included in the article's Creative Commons license and your intended use is not permitted by statutory regulation or exceeds the permitted use, you will need to obtain permission directly from the copyright holder. To view a copy of this license, visit http://creativecommons. org/licenses/by/4.0/.

(c) The Author(s) 2021 